\title{
Molecular diagnosis of diffuse glioma using a chip-based digital PCR system to analyze IDH,TERT, and $H 3$ mutations in the cerebrospinal fluid
}

\author{
Yutaka Fujioka ${ }^{1}$. Nobuhiro Hata ${ }^{1}$ (1) - Yojiro Akagi ${ }^{1}$ - Daisuke Kuga ${ }^{1} \cdot$ Ryusuke Hatae $^{1}$ - Yuhei Sangatsuda ${ }^{1}$. \\ Yuhei Michiwaki ${ }^{1} \cdot$ Takeo Amemiya $^{1} \cdot$ Kosuke Takigawa $^{1} \cdot$ Yusuke Funakoshi ${ }^{1} \cdot$ Aki Sako $^{1} \cdot$ Toru Iwaki $^{2} \cdot$ Koji lihara $^{1}$. \\ Masahiro Mizoguchi ${ }^{1}$
}

Received: 26 October 2020 / Accepted: 16 December 2020 / Published online: 8 January 2021

(c) The Author(s) 2021

\begin{abstract}
Purpose Conventional genetic analyzers require surgically obtained tumor tissues to confirm the molecular diagnosis of diffuse glioma. Recent technical breakthroughs have enabled increased utilization of cell-free tumor DNA (ctDNA) in body fluids as a reliable resource for molecular diagnosis in various cancers. Here, we tested the application of a chip-based digital PCR system for the less invasive diagnosis (i.e., liquid biopsy) of diffuse glioma using the cerebrospinal fluid (CSF).

Methods CSF samples from 34 patients with diffuse glioma were collected from the surgical field during craniotomy. Preoperative lumbar CSF collection was also performed in 11 patients. Extracted ctDNA was used to analyze diagnostic point mutations in IDH1 R132H, TERT promoter (C228T and C250T), and H3F3A (K27M) on the QuantStudio ${ }^{\circledR}$ 3D Digital PCR System. These results were compared with their corresponding tumor DNA samples.

Results We detected either of the diagnostic mutations in tumor DNA samples from 28 of 34 patients. Among them, we achieved precise molecular diagnoses using intracranial CSF in 20 (71\%). Univariate analyses revealed that the World Health Organization (WHO) grade $(\mathrm{p}=0.0034)$, radiographic enhancement $(\mathrm{p}=0.0006)$, and Mib1 index $(\mathrm{p}=0.01)$ were significant predictors of precise CSF-based molecular diagnosis. We precisely diagnosed WHO grade III or IV diffuse gliomas using lumbar CSF obtained from 6 (87\%) of 7 patients with tumors harboring any mutation.

Conclusion We established a novel, non-invasive molecular diagnostic method using a chip-based digital PCR system targeting ctDNA derived from CSF with high sensitivity and specificity, especially for high-grade gliomas.
\end{abstract}

Keywords Cerebrospinal fluid · Chip-based digital PCR · Diffuse glioma $\cdot$ ctDNA $\cdot$ Liquid biopsy

\section{Introduction}

Molecular diagnosis of diffuse gliomas is conventionally performed using surgically obtained tumor tissues; however, the 2016 central nervous system (CNS) World Health Organization (WHO) classification defined a novel tumor [diffuse midline glioma, $H 3 \mathrm{~K} 27 \mathrm{M}$ mutation (DMG, $H 3$ K27M-mut)], diagnosis of which was confirmed by molecular findings [1]. A potential diagnostic issue is that biopsies need to originate from deep tissues, such as the brainstem, and surgery to obtain tissue samples for molecular diagnosis represents a high-risk intervention. Accordingly, the development of less invasive methods for detecting tumor-specific mutations is essential.

Recent progress in genetic analysis technologies such as digital PCR systems allows precise detection of genetic 
alterations from small samples [2,3] and has promoted molecular-diagnostics applications that analyze bodily fluids (liquid biopsy), specifically in various cancers, as a less invasive method for diagnosis and monitoring $[4,5]$. Recent reports revealed the presence of cell-free tumor (ct) DNA in the cerebrospinal fluid (CSF) obtained from patients with CNS tumors [6-8], and ctDNA is also used as a target for liquid biopsy of diffuse glioma [9-11]. This promising approach promotes the utilization of molecular findings as diagnostic markers of diffuse gliomas for monitoring disease state and ultimately the development of non-operative diagnostic procedures. In the present study, we established a novel assay that applies liquid biopsy targeting the ctDNA in CSF using chip-based digital PCR (dPCR) for detecting the glioma-specific diagnostic driver mutations such as $I D H I$ R132H (IDH1), H3F3A K27M (H3 K27M), and TERT promoter (pTERT) mutations. Additionally, we evaluated the feasibility of this assay using CSF samples from patients with diffuse glioma.

\section{Methods}

\section{Study population and sample collection}

Intracranial CSF samples were collected from adult and adolescent patients between July 2017 and November 2019 during surgical resection of intracranial tumors under a preoperative diagnosis of diffuse glioma. We collected 3-10 mL of CSF from the operative field immediately after opening the dura under general anesthesia to prevent contamination with DNA released from the resected tumor during surgical manipulation. We also preoperatively collected $1-6 \mathrm{~mL}$ of CSF by lumbar puncture from enrolled adult and adolescent patients who underwent tumor resection of the intracranial tumor under preoperative diagnosis of diffuse glioma without highly elevated intracranial pressure between March 2019 and February 2020. We excluded non-glioma patients after confirmation of pathological diagnosis.

We centrifuged CSF samples at $5000 \mathrm{~g}$ for $10 \mathrm{~min}$ within $1 \mathrm{~h}$ of collection to remove residual cells and stored them at $-80^{\circ} \mathrm{C}$ as described with slight modification [12]. DNA was extracted from 1 to $3 \mathrm{~mL}$ CSF using the QIAamp circulating nucleic acid kit (Qiagen, Hilden, Germany) according to manufacturer instructions and eluted in a final volume of $60 \mu \mathrm{L}$. Quantification of cell-free DNA (cfDNA) was determined using the Qubit 2.0 fluorimeter with the Qubit dsDNA HS assay kit (Thermo Fisher Scientific, Foster City, CA, USA). Extracted cfDNA was stored at $4{ }^{\circ} \mathrm{C}$ and analyzed within 1 week.

CSF samples were obtained and analyzed in accordance with the Declaration of Helsinki, with the approval of the
Ethics Committee of our institute, and with written consent from patients.

\section{dPCR}

Genotyping of cfDNA and tumor DNA was performed using the QuantStudio ${ }^{\circledR}$ 3D Digital PCR System (Life Technologies, Carlsbad, CA, USA). The DNA template, QuantStudio ${ }^{\circledR}$ master mix, and the assay containing primer/ probe were mixed according to the manufacturer protocol to obtain a dPCR reaction.

The TaqMan dPCR liquid biopsy assay (Life Technologies) was used for pTERT C228T and pTERT C250T analyses, and a custom TaqMan SNP genotyping assay was used for $I D H I$ and $H 3 \mathrm{~K} 27 \mathrm{M}$ mutation analyses. The primer and probe sequence data for each assay are described in the Supplemental Digital Content (see Table 1 in Supplemental Digital Content). The final 14.5- $\mu \mathrm{L}$ dPCR mixture was loaded onto a QuantStudio ${ }^{\circledR}$ 3D Digital PCR chip version2 and subjected to PCR amplification using the QuantStudio ${ }^{\circledR}$ 3D GeneAmp PCR system 9700 (see Table 2 in Supplemental Digital Content). Thermal cycling was performed according to the manufacturer's instructions (see Table 3 in Supplemental Digital Content). Following the reaction, data were analyzed using QuantStudio ${ }^{\circledR}$ 3D Analysis Suite (v.3.1).

\section{Conventional genotyping of tumor-tissue DNA}

We isolated and purified DNA from snap-frozen $\left(-80{ }^{\circ} \mathrm{C}\right)$ intraoperative tumor samples obtained from all patients whose CSF was analyzed, using QIAmp DNA mini kits (Qiagen). We confirmed the presence of IDH1, pTERT $\mathrm{C} 228 \mathrm{~T}$ or $\mathrm{C} 250 \mathrm{~T}$, and $H 3 \mathrm{~K} 27 \mathrm{M}$ point mutations by highresolution melt analysis and subsequent Sanger sequencing, as described previously $[13,14]$. Loss of heterozygosity (LOH) on chromosome $1 \mathrm{p}$ and $19 \mathrm{q}$ was confirmed by a PCRbased LOH assay using microsatellite markers, as described previously [15].

\section{Gel electrophoresis}

Tris-acetate-EDTA ( $\mathrm{pH}$ 8.0) was used as electrophoresis buffer, and a $1.5 \%$ agarose gel was used for the electrophoresis of a sample prepared by mixing $5 \mu \mathrm{L}$ of DNA template with 6X loading buffer (TAKARA, Shiga, Japan). Electrophoresis was performed using a Mupid R-exu system (Mupid Co., Ltd., Tokyo, Japan) at $100 \mathrm{~V}$ for $30 \mathrm{~min}$, followed by staining with EtBr solution and detection and imaging using a Limited-STAGE-UV system (AMZ Systems Science, Osaka, Japan). 


\section{Statistical analysis}

Student's $t$ test was used to compare the DNA concentrations at different collection sites. Associations between ctDNA positivity and patient characteristics were assessed using non-parametric tests, including the Wilcoxon rank-sum test (continuous variables) or Fisher's exact test (categorical variables). All statistical tests were two-sided, with an $\alpha$ value of $\leq 0.05$ used to determine statistical significance. Statistical analysis was performed using JMP software (v.15.0; SAS Institute Japan, Tokyo, Japan).

\section{Results}

\section{Primer design for APCR of cfDNA}

To verify the reproducibility of PCR products amplified from CSF-derived cfDNA, two pairs of cfDNA and the corresponding tumor-tissue DNA obtained from different patients were subjected to gel electrophoresis (Fig. 1). Discrete cfDNA bands at $150 \mathrm{bp}$ and $300 \mathrm{bp}$ (the nucleosome footprint [16]) were clearly visualized exclusively in CSF cfDNA, consistent with previous reports [17]. Taken into consideration that a recent study revealed that fragment size of ctDNA is shorter than cfDNA from normal cells [18], the amplicons of significantly below $150 \mathrm{bp}$ were considered appropriate to ensure stable PCR products when using CSF cfDNA as a template. Therefore, we designed the primers targeting 88- and 107-bp amplicons for IDHI and H3 K27M assays, respectively (see Table 1 in Supplemental Digital Content).

\section{dPCR protocol for cfDNA samples}

Figure 2a shows the plots of cfDNA concentrations in all collected CSF samples obtained from the intracranial operative field and lumbar puncture. The distribution of cfDNA concentrations differed significantly between the two sites, with significantly lower cfDNA concentrations obtained from CSF samples from lumbar puncture relative to those from intraoperative CSF samples $(p=0.0006)$. A direct comparison was performed on four patients having available paired intracranial and lumbar samples, with higher concentrations observed in intracranial samples than in lumbar CSF, except for a sample pair obtained from a patient with the highest class III cytology (Fig. 2b). Notably, the mean cfDNA concentrations in the intracranial and lumbar CSF samples were $73.33 \mathrm{ng} / \mathrm{mL}$ and $7.31 \mathrm{ng} / \mathrm{mL}$, respectively, which were far below the recommended final concentration (1 $\mathrm{ng} / \mu \mathrm{L}$ ) for dPCR templates [3]. However, as described

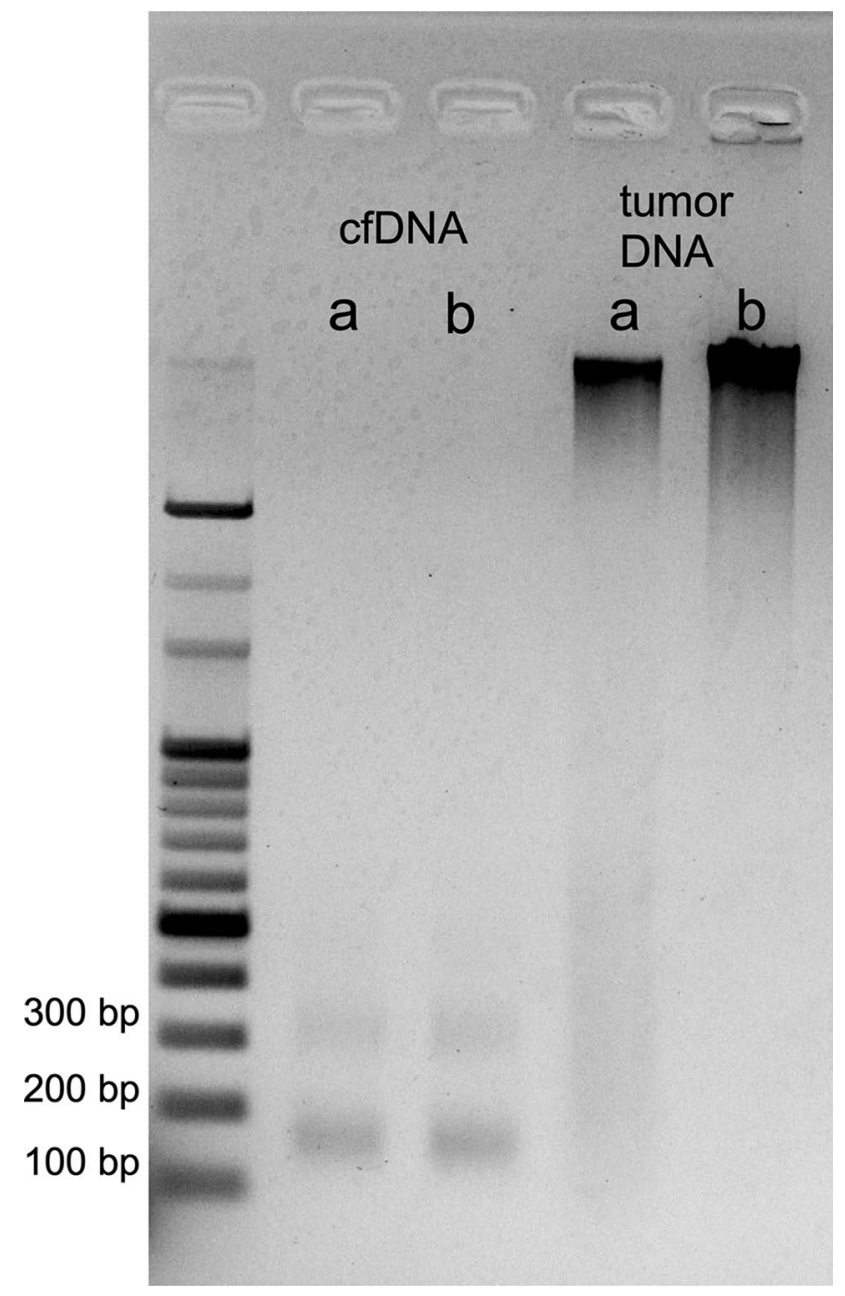

Fig. 1 Agarose gel electrophoresis shows discrete bands at 150 and $300 \mathrm{bp}$ exclusively in lanes of cell-free DNA extracted from cerebrospinal fluid of two patients (left) compared with widespread smear bands for corresponding tumor-tissue DNA (right). Two representative samples show that a high concentration of cell-free (cf)DNA can be extracted from intracranial cerebrospinal fluid. Since the concentration of cfDNA was low, we did not adjust the concentration. a Recurrent anaplastic oligodendroglioma in a 43-year-old female; cfDNA concentration, $4.3 \mathrm{ng} / \mu \mathrm{L}$. b First onset of diffuse midline glioma with $H 3 \mathrm{~K} 27 \mathrm{M}$ in a 46-year-old female; DNA concentration, $4.1 \mathrm{ng} / \mu \mathrm{L}$. Tumor DNA $15.0 \mathrm{ng} / \mu \mathrm{L}$ was loaded

in the next paragraph, we managed to perform digital PCR according to our original protocol, allowing the usage of DNA samples with low concentrations (see Fig. 2a).

\section{Mutation status according to $\mathrm{dPCR}$ results}

For mutation analysis, we used a pair of VIC- and FAMlabeled TaqMan probes annealing to wild type and mutant alleles, respectively [3, 19], and established the threshold values for both VIC and FAM fluorescence using control DNAs. Because the assay needs to tolerate extremely low DNA concentrations, we diluted the negative control 
a

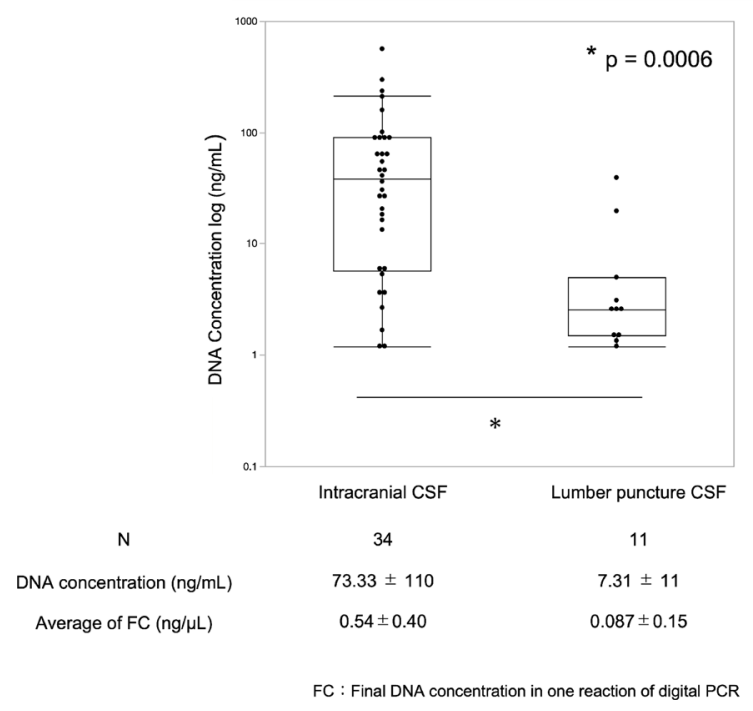

b

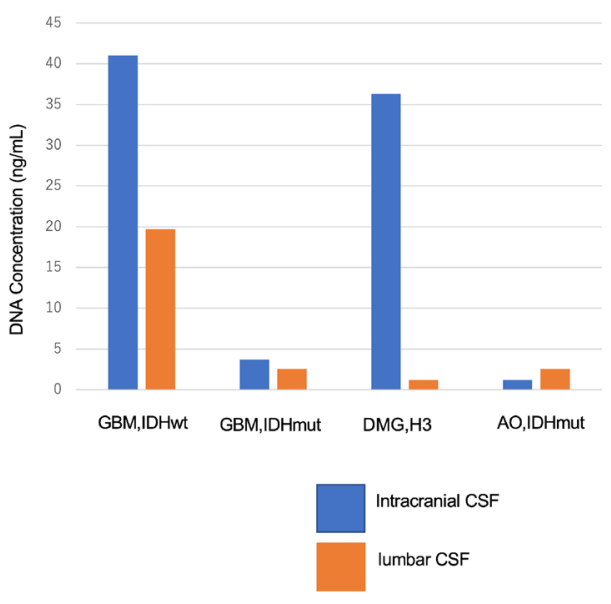

Fig. 2 a Wilcoxon rank-sum test for differences in cell-free DNA concentration between the intracranial cerebrospinal fluid and that obtained after lumbar puncture. b Direct comparison of extracted cell-free DNA concentration between two sites in four patients

(NC) cfDNA obtained from a patient with wild-type (WT) tumor DNA to final reaction concentrations of $1.0 \mathrm{ng} / \mu \mathrm{L}$, $0.1 \mathrm{ng} / \mu \mathrm{L}$, and $0.01 \mathrm{ng} / \mu \mathrm{L}$ each (see Fig. 1 in Supplemental Digital Content). Raw data for each reaction were retrieved and analyzed, and fluorescence values were normalized for homogeneity. We performed quadruplicate $\mathrm{NC}$ experiments (at $1 \mathrm{ng} / \mu \mathrm{L}$ ) to establish threshold values to determine VIC positivity at a certain distance and calculated as the average distance from the peak value of the first distribution and the lowest value of the second distribution apart from the mode point of the VIC histogram. The threshold value for determining FAM positivity was established at a certain distance from the mode point of the FAM histogram and the maximum value of the distributions and excluding sporadic, high-fluorescence spots. The threshold values for all four mutations were established in a similar manner (see Table 4 in Supplemental Digital Content). The scatter plot of fluorescence intensity for each well showed nonspecific VIC and FAM doublepositive plots, even in the NC (see Fig. 1A in Supplemental Digital Content). Theoretically, dPCR performed using very low DNA concentrations involves the distribution of template DNA at less than one copy/well, with mutant DNA detected as FAM-positive and VIC-negative plots. Therefore, we defined positive determination of the presence of mutation(s) as the existence of FAM-positive and VIC-negative plots (see Fig. 1C in Supplemental Digital Content). We performed duplicate analyses of the clinical samples and confirmed the mutation when two or more spots were detected in either of the duplicate results. An example of $I D H$-positive and -negative samples is shown in the Supplemental Digital Content (see Fig. 2 in Supplemental Digital Content).

\section{cfDNA analysis from intracranial CSF}

Among 53 patients from whom intracranial CSF was obtained during surgery, 34 had confirmed, pathologicallydiagnosed, diffuse glioma. Table 1 summarizes the characteristics of these 34 patients, who were grouped according to WHO tumor grades II, III and IV $(n=5,13$ and 16 , respectively).

Supplementary Table 5 shows a comparison of the genotype profiles between dPCR results with CSF cfDNA and Sanger sequencing with the corresponding tumor-tissue DNA. Samples without mutation(s) in the corresponding tumor were determined as WT in CSF cfDNA for $I D H I$ $\mathrm{R} 132 \mathrm{H}(n=16), \mathrm{pTERT} \mathrm{C} 228 \mathrm{~T}(n=22)$ and C250T $(n=32)$, and $H 3 \mathrm{~K} 27 \mathrm{M}(n=32)$ assays. There were no false-positive findings among cfDNA results when tumor-tissue DNA results were used as controls. These results confirmed the high specificity of these assays. Because 28 (82\%) of 34 tumors harbored at least one mutation in tumor-tissue DNA, we evaluated the reliability of dPCR within these results. The genotypes of all glioblastoma (GBM) samples $(n=7)$ were confirmed by using dPCR, with the $H 3 \mathrm{~K} 27 \mathrm{M}$ mutation confirmed in 4 of 5 patients with DMG, H3 K27Mmut. IDH and pTERT co-mutations, a hallmark of oligodendroglioma [20-22], were confirmed in all eight patients with anaplastic oligodendroglioma. The remaining eight gliomas using cfDNA could not be genotyped except for an anaplastic astrocytoma with an $I D H$ mutation. Univariate 
Table 1 Background of patients who provided intracranial cerebrospinal fluid

\begin{tabular}{|c|c|c|c|}
\hline Total & \multicolumn{3}{|c|}{34} \\
\hline Age(yo) & \multicolumn{3}{|c|}{$45 \pm 16$} \\
\hline \multirow{2}{*}{ Sex } & \multicolumn{2}{|c|}{ Male } & \multicolumn{2}{c|}{ Female } \\
\cline { 2 - 4 } & \multicolumn{2}{|c|}{$17(50 \%)$} & \multicolumn{2}{c|}{$17(50 \%)$} \\
\hline \multirow{2}{*}{ WHO grade } & Grade II & Grade III & GradeIV \\
\cline { 2 - 4 } & $5(15 \%)$ & $13(38 \%)$ & $16(47 \%)$ \\
\hline IDH1 R132H mutation & \multicolumn{3}{|c|}{$16(47 \%)$} \\
\hline TERT promoter C228T mutation & \multicolumn{3}{|c|}{$13(38 \%)$} \\
\hline TERT promoter C250T mutation & \multicolumn{3}{|c|}{$3(9 \%)$} \\
\hline H3F3A K27M mutation & \multicolumn{3}{|c|}{$15(44 \%)$} \\
\hline Recurrence & \multicolumn{3}{|c|}{$38(82 \%)$} \\
\hline Gd enhancement & \multicolumn{3}{|c|}{$7(21 \%)$} \\
\hline Lesion touches CSF space in FLAIR & \multicolumn{3}{|c}{} \\
\hline Radiographical dissemination & \multicolumn{3}{|c|}{} \\
\hline
\end{tabular}

analyses revealed that the WHO grade $(\mathrm{p}=0.0034)$, radiographic enhancement $(\mathrm{p}=0.0006)$, recurrence $(\mathrm{p}=0.0084)$,

Table 2 Univariate analysis

\begin{tabular}{llll}
\hline & $\begin{array}{l}\text { Matched } \\
\mathrm{N}=20\end{array}$ & $\begin{array}{l}\text { Failure } \\
\mathrm{N}=8\end{array}$ & $\mathrm{p}$ \\
\hline WHO grade III or IV & 20 & 4 & 0.0034 \\
Gd enhancement & 20 & 3 & 0.0006 \\
Recurrence & 12 & 0 & 0.0084 \\
Mib-1index(\%) & $33 \pm 21$ & $12 \pm 13$ & 0.01 \\
Radiographical dissemination & 5 & 1 & 0.64 \\
Final concentration $(\mathrm{ng} / \mu \mathbf{L})$ & $0.58 \pm 0.12$ & $0.50 \pm 0.10$ & 0.58 \\
\hline
\end{tabular}

and Mib1 index $(\mathrm{p}=0.01)$ were significant predictive factors for precise CSF-based molecular diagnosis; however, DNA concentration and radiographic dissemination were not predictive (Table 2).

\section{Analysis of cfDNA from CSF obtained by lumbar puncture}

Of the 16 patients undergoing pre-surgery lumbar puncture, pathological diagnosis of diffuse glioma was confirmed in 11 , which were enrolled for subsequent cfDNA genotyping (Table 3). Nine of 11 tumors (82\%) harbored at least one mutation in tumor-tissue DNA, and genotyping results for CSF and tumor tissue matched in all WHO grade IV tumors. In the remaining five cases, accurate results were obtained for two cases: a primary anaplastic astrocytoma involving $I D H$ mutation without radiographic enhancement and a recurrent anaplastic oligodendroglioma manifesting radiographic enhancement and dissemination. The results showed that $6(87 \%)$ of 7 WHO grades III and IV gliomas were precisely diagnosed preoperatively, whereas neither of the grade II tumors could be preoperatively diagnosed.
Similar to results obtained from intracranial CSF, lumbar CSF samples did not return false-positive results during genotyping. Notably, grade IV tumors were accurately diagnosed preoperatively, even in patients with cytology class I or without radiographic dissemination (see Fig. 3 in Supplemental Digital Content).

\section{Discussion}

In this study, we describe the establishment of a novel method for liquid biopsy targeting the ctDNA in CSF using chip-based dPCR to detect glioma-specific diagnostic driver mutations. The 2016 CNS WHO classification and subsequent cIMPACT-NOW update incorporated various gliomaspecific genetic alterations in order to integrate molecular diagnoses [1, 20, 21, 23, 24]. Among these alterations, the 2016 CNS WHO classification regards the IDH mutation and the $1 \mathrm{p} 19 \mathrm{q}$ co-deletion as essential markers for a molecular diagnosis of diffuse glioma, and the $H 3 \mathrm{~K} 27 \mathrm{M}$ mutation as an essential marker of pediatric glioma [1]. Additionally, $\mathrm{pTERT}$ mutation is recognized as a novel essential diagnostic marker based on its presence in GBMs and oligodendrogliomas [22]. Recent studies revealed pTERT mutations as the most prevalent molecular markers detected in $I D H$-WT GBMs and predictive of aggressive bioactivity of diffuse astrocytomas [25]. cIMPACT-NOW (update 3) reached a consensus on designating $I D H$-WT diffuse or anaplastic astrocytomas with pTERT mutation as WHO grade IV [22, 26]. In $I D H$-mutant oligodendroglial tumors, the status of $1 \mathrm{p} 19 \mathrm{q}$ and $\mathrm{p} T E R T$ are strongly correlated, with these markers interchangeable when combined with $I D H$ status [20-22]. Although DMG, H3 K27M-mut predominantly occurs in children and adolescents, high-grade thalamic gliomas from young adults frequently harbor $H 3$ F3A-K27M [27]. The present study of six patients with $H 3$ K27M-mut included three adult-onset cases. Moreover, the frequency of elderly patients with DMG, H3 K27M-mut is notable [28, 29]. We recently described an elderly patient with the DMG, H3 K27M-mut that mimicked a hemispheric malignant glioma [30]. Taken together, the $H 3$ status of an adult hemispheric diffuse glioma affecting the midline should be confirmed even in elderly patients. It is conceivable that the majority of diffuse gliomas can be molecularly diagnosed by confirming $I D H, H 3 \mathrm{~K} 27 \mathrm{M}$, and pTERT status. In the present study, we selected these genes based on this interpretation and also their status as hotspot point mutations, which enable their detection by dPCR via design of a single TaqMan probe per mutation.

Although other liquid biopsy methods for glioma diagnosis exist, sensitivity is essential for selection of analytical methods for future clinical applications. The chip-based dPCR method presented here yielded highly sensitive results 


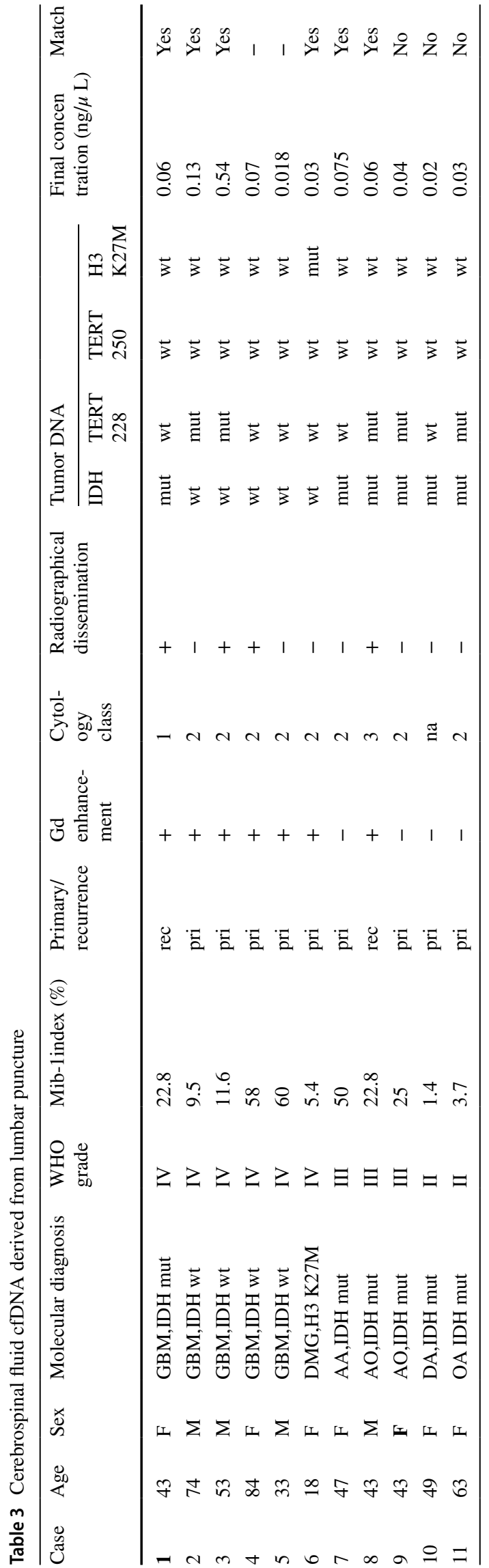

using lumbar puncture CSF, especially for diagnosis highgrade diffuse gliomas. Pentsova et al. [31] collected preoperative CSF by lumbar puncture from 53 patients with suspected brain tumors and detected ctDNA in $6(50 \%)$ of 12 of them with diffuse glioma using next generation sequencing (NGS). Miller et al. [32] also used NGS to analyze CSF samples from patients with diffuse gliomas when a clinical event occurred after completing adjuvant therapy, and identified at least one glioma-related genetic alteration in 42 (49.2\%) of 85 tumors. Although the target genes and backgrounds differed from those in the present study, our results demonstrated a higher sensitivity in the dPCR-based method than NGS-based method.

Currently, commercially available dPCR systems are theoretically divided into droplet digital (dd) PCR and chipbased dPCR. The sensitivity of ddPCR exceeds that of NGS [33]. Martinez et al. [10] tested the capability of ddPCR using cfDNA samples from 20 cases of diffuse gliomas and successfully detected ctDNAs, such as pTERT, IDH, and H3 K27M, in 15 (88\%) of 17 patients using CSF collected from lumbar puncture. This indicated that ddPCR showed comparable or higher sensitivity relative to the APCR assay in the present study; however, Martinez et al. [10] used their method exclusively to validate an already confirmed mutation. Because we performed all four genotyping assays using a limited amount of CSF from each patient, we were forced to analyze further diluted DNAs from the originally extracted samples. Therefore, we speculate that the sensitivity of our results might have been reduced due to the study design, and that the sensitivity could have been improved by focusing on a target gene. Moreover, chip-based dPCR offers a higher degree of cost-effectiveness and simpler operation relative to ddPCR as a practical method [3, 19]. Given the aim of liquid biopsy as allowing molecular diagnosis in the absence of surgery, high specificity is required for clinical applications. These results showed no false-positive determinations of mutations using cfDNAs relative to tumortissue DNA, indicating the extremely high specificity of the method. Accordingly, these findings suggest the efficacy of this assay for future development of a liquid biopsy acceptable for daily clinical use.

One of the major limitations of the present study is that the reliability of our method seemed to depend on the extent of CSF involvement in tumor distribution. Therefore, our method might be most appropriate for diagnosing advanced high-grade glioma involving CSF. However, we should take into consideration that lumbar puncture is not always indicated for patients with advanced high-grade glioma, because of risk of herniation by space-occupying lesions. These issues are associated with another limitation of the present study that most of our samples were obtained by craniotomy, and lumbar CSF was collected from a limited number of our patients. We detected diagnostic mutations in 
28 of 34 patients with glioma using intracranial CSF and the analyzed genes in the remaining six gliomas did not harbor any diagnostic mutations even in tumor tissues. This issue is associated with another limitation that gliomas cannot always be differentially diagnosed from non-glial tumors through genotyping of diagnostic mutations. The sensitivity of our method is lower for lumbar than for intracranial CSF is another limitation. The sensitivity of our method needs to be improved, even for lumbar CSF, before less-invasive liquid biopsies could be routinely used for diagnostic purposes.

\section{Conclusions}

Here, we showed that molecular analysis of diffuse glioma can be performed using chip-based dPCR of ctDNA extracted from patient CSF. Lumber CSF analysis is a less invasive than conventional molecular diagnostic methods. This approach facilitates further development of novel methods of molecular diagnosis and future paradigm shifts in treatment strategies for and clinical management of glioma.

Funding This work was supported by the Japanese Society for the Promotion of Science Grants-in-Aid for Scientific Research (KAKENHI; Nos. 20K09392, 19K17673, 18K08970, 17K16652, 16K10779, and 15K15529), Fujita Memorial Fund for Medical Research(GAKF800362) and Ichiro Kanehara Foundation for the Promotion of Medical Sciences and Medical Care(GAKF800381).

\section{Compliance with ethical standards}

Conflicts of interest The authors declare no actual or potential conflicts of interest.

Ethical approval All CSF samples were collected and analyzed in accordance with the Declaration of Helsinki (2013) and under the approval of the Ethics Committee at the Graduate School of Medical Sciences, Kyushu University (Approval No: 637-04 and 30-332). All patients provided written consent to participate in the study.

Open Access This article is licensed under a Creative Commons Attribution 4.0 International License, which permits use, sharing, adaptation, distribution and reproduction in any medium or format, as long as you give appropriate credit to the original author(s) and the source, provide a link to the Creative Commons licence, and indicate if changes were made. The images or other third party material in this article are included in the article's Creative Commons licence, unless indicated otherwise in a credit line to the material. If material is not included in the article's Creative Commons licence and your intended use is not permitted by statutory regulation or exceeds the permitted use, you will need to obtain permission directly from the copyright holder. To view a copy of this licence, visit http://creativecommons.org/licenses/by/4.0/.

\section{References}

1. Louis DN, Perry A, Reifenberger G et al (2016) The 2016 World Health Organization classification of tumors of the central nervous system: a summary. Acta Neuropathol 131(6):803-820. https ://doi.org/10.1007/s00401-016-1545-1

2. Hindson BJ, Ness KD, Masquelier DA et al (2011) Highthroughput droplet digital PCR system for absolute quantitation of DNA copy number. Anal Chem 83(22):8604-8610. https://doi. org/10.1021/ac202028g

3. Majumdar N, Wessel T, Marks J (2015) Digital PCR modeling for maximal sensitivity, dynamic range and measurement precision. PLoS ONE 10(3):1-17. https://doi.org/10.1371/journ al.pone. 0118833

4. Siravegna G, Marsoni S, Siena S, Bardelli A (2017) Integrating liquid biopsies into the management of cancer. Nat Rev Clin Oncol 14(9):531-548. https://doi.org/10.1038/nrclinonc.2017.14

5. Gorgannezhad L, Umer M, Islam MN, Nguyen NT, Shiddiky MJA (2018) Circulating tumor DNA and liquid biopsy: opportunities, challenges, and recent advances in detection technologies. Lab Chip 18(8):1174-1196. https://doi.org/10.1039/c81c00100f

6. Wang Y, Springer S, Zhang $M$ et al (2015) Detection of tumorderived DNA in cerebrospinal fluid of patients with primary tumors of the brain and spinal cord. Proc Natl Acad Sci USA 112(31):9704-9709. https://doi.org/10.1073/pnas.1511694112

7. Pan W, Gu W, Nagpal S, Gephart MH, Quake SR (2015) Brain tumor mutations detected in cerebral spinal fluid. Clin Chem 61(3):514-522. https://doi.org/10.1373/clinchem.2014.235457

8. Hiemcke-Jiwa LS, Minnema MC, Radersma-van Loon JH et al (2018) The use of droplet digital PCR in liquid biopsies: a highly sensitive technique for MYD88 p.(L265P) detection in cerebrospinal fluid. Hematol Oncol 36(2):429-435. https://doi.org/10.1002/ hon. 2489

9. Huang TY, Piunti A, Lulla RR et al (2017) Detection of histone H3 mutations in cerebrospinal fluid-derived tumor DNA from children with diffuse midline glioma. Acta Neuropathol Commun 5(1):1-12. https://doi.org/10.1186/s40478-017-0436-6

10. Martínez-Ricarte F, Mayor R, Martínez- Sáez E et al (2018) Molecular diagnosis of diffuse gliomas through sequencing of cell-free circulating tumor DNA from cerebrospinal fluid. Clin Cancer Res 24(12):9-11. https://doi.org/10.1158/1078-0432. ccr-17-3800

11. Juratli TA, Stasik S, Zolal A et al (2018) TERT promoter mutation detection in cell-free tumor-derived DNA in patients with IDH wild-type glioblastomas: a pilot prospective study. Clin Cancer Res 24(21):5282-5291. https://doi.org/10.1158/1078-0432. ccr-17-3717

12. Juratli TA, Stasik S, Zolal A et al (2018) TERT promoter mutation detection in cell-free tumor-derived DNA in patients with IDH wild-type glioblastomas: a pilot prospective study. Clin Cancer Res. https://doi.org/10.1158/1078-0432.ccr-17-3717

13. Hatae R, Hata N, Yoshimoto K et al (2016) Precise detection of IDH1/2 and BRAF hotspot mutations in clinical glioma tissues by a differential calculus analysis of high-resolution melting data. PLoS ONE 11(8):1-16. https://doi.org/10.1371/journ al.pone.0160489

14. Hata N, Yoshimoto K, Hatae R et al (2017) Add-on bevacizumab can prevent early clinical deterioration and prolong survival in newly diagnosed partially resected glioblastoma patients with a poor performance status. Onco Targets Ther 10:429-437. https:// doi.org/10.2147/ott.s125587

15. Ma X, Yoshimoto K, Guan Y et al (2012) Associations between microRNA expression and mesenchymal marker gene expression in glioblastoma. Neuro Oncology 14(9):1153-1162. https://doi. org/10.1093/neuonc/nos145 
16. Snyder MW, Kircher M, Hill AJ, Daza RM, Shendure J (2016) Cell-free DNA comprises an in vivo nucleosome footprint that informs its tissues-of-origin. Cell 164(1-2):57-68. https://doi. org/10.1016/j.cell.2015.11.050

17. Mouliere F, Mair R, Chandrananda D et al (2018) Detection of cell-free DNA fragmentation and copy number alterations in cerebrospinal fluid from glioma patients. EMBO Mol Med 10(12):2016. https://doi.org/10.15252/emmm.201809323

18. Guo J, Ma K, Bao H et al (2020) Quantitative characterization of tumor cell-free DNA shortening. BMC Genom 21:473. https:// doi.org/10.1186/s12864-020-06848-9

19. Pohl G, Shih I (2004) Principle and applications of digital PCR. Expert Rev Mol Diagn 4(1):41-47. https://doi.org/10.1586/14737 159.4.1.41

20. Louis DN, Wesseling P, Paulus W et al (2018) cIMPACT-NOW update 1: not otherwise specified (NOS) and not elsewhere classified (NEC). Acta Neuropathol 135(3):481-484. https://doi. org/10.1007/s00401-018-1808-0

21. Louis DN, Giannini C, Capper D et al (2018) cIMPACT-NOW update 2: diagnostic clarifications for diffuse midline glioma, H3K27M-mutant and diffuse astrocytoma/anaplastic astrocytoma, IDH-mutant. Acta Neuropathol 135(4):639-642. https:// doi.org/10.1007/s00401-018-1826-y

22. Arita H, Narita Y, Fukushima S et al (2013) Upregulating mutations in the TERT promoter commonly occur in adult malignant gliomas and are strongly associated with total $1 \mathrm{p} 19 \mathrm{q}$ loss. Acta Neuropathol 126(2):267-276. https://doi.org/10.1007/s0040 1-013-1141-6

23. Brat DJ, Aldape K, Colman H et al (2018) cIMPACT-NOW update 3: recommended diagnostic criteria for "Diffuse astrocytic glioma, IDH-wildtype, with molecular features of glioblastoma, WHO grade IV". Acta Neuropathol 136(5):805-810. https://doi. org/10.1007/s00401-018-1913-0

24. Ellison DW, Hawkins C, Jones DTW et al (2019) cIMPACT-NOW update 4: diffuse gliomas characterized by MYB, MYBL1, or FGFR1 alterations or BRAF V600E mutation. Acta Neuropathol 137(4):683-687. https://doi.org/10.1007/s00401-019-01987-0

25. Pekmezci M, Rice T, Molinaro AM et al (2017) Adult infiltrating gliomas with WHO 2016 integrated diagnosis: additional prognostic roles of ATRX and TERT. Acta Neuropathol 133(6):1001-1016. https://doi.org/10.1007/s00401-017-1690-1

26. Louis DN, Wesseling P, Aldape K et al (2020) cIMPACT-NOW update 6: new entity and diagnostic principle recommendations of the cIMPACT-Utrecht meeting on future CNS tumor classification and grading. Brain Pathol 30(4):844-856. https://doi.org/10.1111/ bpa. 12832

27. Aihara K, Mukasa A, Gotoh K et al (2014) H3F3A K27M mutations in thalamic gliomas from young adult patients. Neuro Oncology $16: 140-146$

28. Solomon DA, Wood MD, Tihan T et al (2016) Diffuse midline gliomas with histone H3-K27M mutation: a series of 47 cases assessing the spectrum of morphologic variation and associated genetic alterations. Brain Pathol 26:569-580

29. Schreck KC, Ranjan S, Skorupan N et al (2019) Incidence and clinicopathologic features of H3 K27M mutations in adults with radiographically determined midline gliomas. J Neurooncol 143:87-93

30. Fujioka Y, Hata N, Hatae R et al (2020) A case of diffuse midline glioma, H3 K27M mutant mimicking a hemispheric malignant glioma in an elderly patient. Neuropathology 40:99-103. https:// doi.org/10.1111/neup.12609

31. Pentsova EI, Shah RH, Tang J et al (2016) Evaluating cancer of the central nervous system through next-generation sequencing of cerebrospinal fluid. J Clin Oncol 34(20):2404-2415. https://doi. org/10.1200/jco.2016.66.6487

32. Miller AM, Shah H, Pentsova I et al (2019) Tracking tumour evolution in glioma through liquid biopsies of cerebrospinal fluid. Nature 565:654-658. https://doi.org/10.1038/s41586-019-0882-3

33. Saenz-Antoñanzas A, Auzmendi-Iriarte J, Carrasco-Garcia E et al (2019) Liquid biopsy in glioblastoma: opportunities, applications and challenges. Cancers (Basel) 1(7):1-20. https://doi. org/10.3390/cancers 11070950

Publisher's Note Springer Nature remains neutral with regard to jurisdictional claims in published maps and institutional affiliations. 